\title{
Liver Disease and Hemostatic (Dys)function
}

\author{
Armando Tripodi, $\mathrm{PhD}^{1}$ \\ ${ }^{1}$ Department of Clinical Sciences and Community Health, Università \\ degli Studi di Milano and IRCCS Cà Granda Ospedale Maggiore \\ Foundation, Milano, Italy
}

Address for correspondence Armando Tripodi, PhD, Via Pace 9, 20122-Milano, Italy (e-mail: armando.tripodi@unimi.it).

Semin Thromb Hemost 2015;41:462-467.

\begin{abstract}
Keywords

- cirrhosis

- thrombin generation

- hemorrhage

- thrombosis

Cirrhosis presents with decreased procoagulant factors as a consequence of the impaired synthetic capacity of the liver. This was taken as evidence to explain the abnormalities of the coagulation tests prothrombin time (PT) and activated partial thromboplastin time (aPTT) and the bleeding events that occur in these patients. It was for long time (and probably is still) common practice to test patients with the PT and to treat those with predefined (but arbitrary) cutoff values with plasma or prohemostatic agents to prevent or stop bleeding. However, anticoagulant factors that contrast the procoagulants are also decreased in cirrhosis. It was therefore postulated that the coagulation balance (i.e., the net result between the action of pro- and anticoagulants) is somewhat rebalanced. Subsequent studies supported this view showing that plasma from cirrhotic patients generates normal amounts of thrombin. In addition, primary hemostasis (i.e., platelet-vessel wall interaction) is rebalanced notwithstanding cirrhosis present with thrombocytopenia. It was shown that increased levels of the adhesive protein von Willebrand factor (a typical feature of cirrhosis) compensate for the low number (function) of platelets. The earlier considerations have been instrumental to help dismantle the old paradigms of cirrhosis as the epitome of the acquired hemorrhagic coagulopathies and the traditional coagulation tests PT and aPTT as suitable predictors of bleeding risk. The demise of the old paradigms and the rise of the new one may have important practical implications for the management of patients with cirrhosis and will be discussed in this chapter.
\end{abstract}

The liver synthesizes circulating coagulation proteins needed for the complex hemostatic process that operates in vivo to stop bleeding and prevent the unwanted thrombin generation and fibrin deposition. As a consequence, liver dysfunction is invariably associated with decreased levels of plasma coagulation proteins (except for factor VIII and von Willebrand factor, which are increased). ${ }^{1}$ These observations have been taken over the years as evidence to explain the abnormalities of traditional coagulation tests such as the prothrombin time (PT) and activated partial thromboplastin time (aPTT) that are typically observed in patients with cirrhosis, but also to explain the bleeding events that are occasionally associated with this condition. While the first is reasonable, the second needs comments and reconsideration. This article aims at discussing observations provided by the most recent literature that makes the long-lasting belief of chronic liver disease as the prototype of the acquired hemorrhagic coagulopathies to be reconsidered and an old paradigm to be dismantled. This change of paradigm will have profound practical impact on how the complex hemostatic dysfunction is managed in this category of patients. The key points and messages of the article are summarized in - Table $\mathbf{1}$.

Hemostasis in vivo is a tightly regulated mechanism responsible for the conversion of fibrinogen into fibrin and its subsequent degradation. The entire mechanism can be dissected into the following three parts: platelet-vessel wall interaction (also known as primary hemostasis), coagulation (i.e., thrombin generation and fibrin formation), and fibrin published online June 16, 2015
Issue Theme Hemostatic Dysfunction in Liver Diseases; Guest Editors: Ton Lisman, PhD, and Hau C. Kwaan, MD, FRCP.
Copyright $\odot 2015$ by Thieme Medical Publishers, Inc., 333 Seventh Avenue, New York, NY 10001, USA. Tel: +1(212) 584-4662.
DOI http://dx.doi.org/ $10.1055 / \mathrm{s}-0035-1550440$. ISSN 0094-6176. 
Table 1 Hemostatic dysfunction in chronic liver disease ${ }^{a}$

\begin{tabular}{|c|c|}
\hline Platelet count & Decreased $^{2}$ \\
\hline von Willebrand factor & Increased $^{4}$ \\
\hline (Primary) hemostasis balance & $\begin{array}{l}\text { Restored because of the increased levels of the adhesive protein von Willebrand } \\
\text { factor } 4\end{array}$ \\
\hline $\begin{array}{l}\text { Procoagulant factors other than FVIII and } \\
\text { von Willebrand factor }\end{array}$ & Decreased $^{1}$ \\
\hline FVIII & Increased $^{28}$ \\
\hline Anticoagulant factors & Decreased $^{1}$ \\
\hline Coagulation balance & Restored because of the concomitant deficiency of pro- and anticoagulants ${ }^{10}$ \\
\hline PT and aPTT & $\begin{array}{l}\text { Prolonged, but they do not represent the mechanism of coagulation operating } \\
\text { in vivo. }{ }^{17} \text { They are responsive to the procoagulants, but much less to the } \\
\text { anticoagulants. Their abnormalities are poorly associated with bleeding }{ }^{12}\end{array}$ \\
\hline The balance of hemostasis in cirrhosis & $\begin{array}{l}\text { Although restored is unstable because of the relative deficit of pro- and } \\
\text { anticoagulants as well as thrombocytopenia. It may tip toward hemorrhage or } \\
\text { thrombosis, depending on the circumstantial risk factors or conditions }\end{array}$ \\
\hline $\begin{array}{l}\text { Conditions associated with cirrhosis that } \\
\text { may explain bleeding }\end{array}$ & Portal hypertension, bacterial infections, endothelial dysfunction, renal failure \\
\hline Thrombosis in cirrhosis & $\begin{array}{l}\text { Patients with cirrhosis are not fully protected from venous thromboembolism } \\
\text { (VTE). }{ }^{24} \text { Their relative risk for VTE has been estimated two times higher than that } \\
\text { of noncirrhotic patients. }{ }^{25}\end{array}$ \\
\hline $\begin{array}{l}\text { Consequences stemming from the } \\
\text { aforementioned considerations }\end{array}$ & $\begin{array}{l}\text { - PT and aPTT should not be used as predictors of bleeding or to guide transfusion } \\
\text { with fresh frozen plasma or procoagulant agents. } \\
\text { - Infusion of plasma or procoagulant agents is poorly effective in controlling } \\
\text { bleeding }{ }^{13-16} \text { and may occasionally result in adverse events. Their } \\
\text { indiscriminate use should be discouraged. } \\
\text { - The same considerations apply to platelet transfusion or treatment with } \\
\text { agonists of the thrombopoietin receptors } 7,8 \\
\text { - Patients with cirrhosis are not auto-anticoagulated and deserve therefore to be } \\
\text { treated with anticoagulants to treat or prevent thrombosis. }\end{array}$ \\
\hline
\end{tabular}

Abbreviations: aPTT, activated partial thromboplastin time; PT, prothrombin time.

${ }^{\mathrm{a} K e y}$ points only; see the text for details.

degradation (i.e., fibrinolysis). In the following paragraphs, two of the three mechanisms will be revisited. Fibrinolysis will be discussed in another chapter of this journal.

\section{Platelet-Vessel Wall Interaction}

Platelets contribute to the hemostatic process by adhesion to the subendothelial matrix at the site of vessel wall injury by means of the adhesive multimeric protein von Willebrand factor. On adhesion, they undergo activation, shape change, and secretion that ultimately lead to aggregation. In a subsequent step, they express the negatively charged phospholipid phosphatidylserine on their surface, which acts as a receptor for the vitamin $\mathrm{K}$-dependent coagulation factors needed to speed up thrombin generation, ultimately leading to the conversion of fibrinogen into fibrin. As a consequence of the variable thrombocytopenia that is associated with cirrhosis, ${ }^{2}$ it is generally believed that the function of platelets in this setting is hampered, and that platelet counts should be restored to prevent or stop bleeding before or during invasive procedures. ${ }^{3}$ This concept is based on biological plausibility and much less on evidence stemming from clinical trials. Furthermore, neither the platelet count that would indicate the need for infusion nor the target platelet numbers are known. Finally, hemostatic tests that are able to reliably guide transfusion are not widely available and the significance of their results are not precisely known.

Recently, it was shown that patients with cirrhosis display platelet adhesion in a flowing system (mimicking the conditions that occur in vivo) similar to those of healthy subjects, despite these patients being thrombocytopenic. Apparently, high levels of von Willebrand factor, typically observed in these patients, compensate for the low number (and function) of platelets. ${ }^{4}$ More recently, it was demonstrated that also thrombin generation in platelet-rich plasma in cirrhosis is normal if platelet numbers are higher than $60 \times 10^{9} / \mathrm{L}^{5}$ The earlier observations can be taken as indications that patients with cirrhosis do not need an indiscriminate platelet transfusion unless they are severely thrombocytopenic. A recent study demonstrated that infusion of one single adult platelet unit was not able to substantially increase platelet numbers of patients with cirrhosis with preinfusion values of $50 \times 10^{9} / \mathrm{L}$ or less. ${ }^{6}$ Moreover, neither thrombin generation in plateletrich plasma nor thromboelastometry parameters in whole blood were significantly altered by the transfusion. ${ }^{6}$ These observations seem to indicate that for a substantial platelet 
increase multiple transfusions are required. As an alternative, an increased platelet count could be obtained with treatment with agonists of the thrombopoietin receptors such as eltrombopag. ${ }^{6}$ It should, however, be realized that a recent clinical trial on the efficacy of eltrombopag to increase the platelets count in patients with cirrhosis undergoing invasive procedures, was prematurely terminated because of an excessive rate of thrombotic events in the eltrombopag arm of the study. ${ }^{7}$ All in all, the earlier observations indicate that decision on whether platelets should be increased in individual patients should not be made indiscriminately, but after careful consideration of the risk/benefit ratio. ${ }^{8}$

\section{Coagulation}

As aforementioned, coagulation is a tightly regulated mechanism in which the procoagulant drivers (i.e., procoagulant proteins) speed up thrombin generation to convert fibrinogen into fibrin. The procoagulant drivers are opposed in vivo by their anticoagulant counterparts, that is, the naturally occurring anticoagulants antithrombin, protein C, protein S, and the tissue factor pathway inhibitor. Under physiological conditions, the two drivers oppose each other and prevent the unwanted thrombin generation and fibrin deposition. Like the procoagulants, the anticoagulants are also synthesized by the liver and consequently the pro- and anticoagulants are concomitantly decreased in patients with cirrhosis. The pathophysiological significance of this simple observation escaped the attention of clinicians for many years and very few realized that there might be a rebalanced coagulation in patients with chronic liver disease. ${ }^{9}$ In 2005 , support to the earlier postulate was provided by an in vitro study showing that thrombin generation in patients with cirrhosis is essentially normal if investigated by global tests responsive to both the pro- and anticoagulants. ${ }^{10}$ Thrombin generation tests can be made responsive to both pro- and anticoagulants by the addition of thrombomodulin, thus allowing full activation of the protein $C$ anticoagulant system. ${ }^{11}$ Thrombomodulin is located on endothelial cells and much less in plasma.

The PT and aPTT are coagulation tests with clot formation as an endpoint that occur few seconds after the activation of coagulation. Importantly, they are performed using plasma in the absence of thrombomodulin. All these considerations lead to the logical conclusion that PT and aPTT are responsive to the procoagulant drivers (they are in fact perfectly suitable to diagnose hemophilia and allied disorders), but much less to the anticoagulant drivers (they are in fact normal in patient with congenital deficiency of the naturally occurring anticoagulants). This explains the poor correlation between bleeding and the abnormality of these tests that has been observed but not underscored for many years in patients with cirrhosis. $^{12}$ This notwithstanding, the use of PT and aPTT as predictors of bleeding is still common practice in cirrhosis, and patients with test results earlier predefined (but arbitrary) cutoff values are frequently given prophylaxis with fresh frozen plasma or prohemostatic agents before invasive procedures.
Recently, clinical trials comparing usual care versus infusion of recombinant activated factor VII (NovoSeven, Novo Nordisk, Bagsvaerd, Denmark; one of the most potent prohemostatic agents) have been performed in patients with cirrhosis with active variceal bleeding ${ }^{13,14}$ (one of the most common and severe bleeding events in cirrhosis) or in patients with cirrhosis undergoing hepatectomy or liver transplant surgery. ${ }^{15,16}$ Results of these trials showed that NovoSeven, while effective in normalizing the PT, ${ }^{13}$ had no effect on bleeding. As a matter of fact, if thrombin generation in cirrhosis is normal, it does not need to be increased by infusion of procoagulant agents. As an alternative to Novo Seven, fresh frozen plasma is often used as prophylaxis in patients with cirrhosis. However, no data from controlled clinical trials are available to determine the efficacy/safety of infusion of fresh frozen plasma in patients with cirrhosis.

Recently, plasma from patients with compensated cirrhosis has been spiked in vitro with appropriate amounts of a pooled normal plasma in a patient-to-normal plasma ratio mimicking an infusion of $15 \mathrm{~mL} / \mathrm{kg}$ fresh frozen plasma ${ }^{17}$ (i.e., the dose commonly used for patient treatment). Mixtures were tested for PT, aPTT, and thrombin generation. Results demonstrated that the PT and aPTT that were abnormally prolonged in the majority of patient plasmas before mixing, were shortened to a considerable degree, but they did not normalize on mixing with the pooled normal plasma. ${ }^{17}$ Conversely, thrombin generation in the presence of thrombomodulin that was within the normal range in all plasmas before mixing, remained unchanged after mixing. ${ }^{16}$ These results seriously question the validity of the PT as a test to guide transfusion of fresh frozen plasma in the setting of cirrhosis.

Although randomized controlled trials with clinical endpoints are needed to substantiate the earlier hypothesis, it is likely that fresh frozen plasma at the dose commonly employed (i.e., $15 \mathrm{~mL} / \mathrm{kg}$ ) can hardly be effective in increasing thrombin generation. Nevertheless, it cannot be excluded that plasma infusion at higher doses, by increasing both the pro- and anticoagulant drivers, could be effective in stabilizing the (the rather unstable) balance of coagulation in these patients. However, this can be likely achieved only by infusing such an amount of plasma resulting in fluid overload and exacerbation of portal hypertension and bleeding. The earlier observations once again support the concept that the PT and aPTT are in vitro tests not suitable to evaluate the coagulation derangement occurring in cirrhosis (and perhaps in other acquired hemorrhagic coagulopathies) $)^{18}$ and should therefore not be used as predictors of bleeding in this setting.

On the contrary, the PT test still maintains its validity as a prognostic index to judge the severity of liver dysfunction. It is incorporated into the equation defining the model of endstage liver disease (MELD) score that is used to prognosticate survival and prioritize patients for liver transplantation. PT results can be expressed as ratio (patient-to-normal clotting times), the higher the ratio the longer the PT or as international normalized ratio (INR). The latter expression is essentially the PT ratio corrected for a sensitivity index 
(international sensitivity index, ISI) relative to a WHO standard thromboplastin (i.e., INR $\left.=\left[\mathrm{PT}_{\text {patient }} / \mathrm{PT}_{\text {normal }}\right]^{\mathrm{ISI}}\right) .{ }^{19}$

The INR has been developed to be used for patients on vitamin $\mathrm{K}$ antagonists (VKA). The coagulation defect induced by VKA on the same patient is judged differently by laboratories using different commercial thromboplastins when the results are expressed as PT ratio. However, the INR proved effective in better harmonizing the results, thus giving patients the opportunity to move freely from one laboratory to another and clinicians to use "universal therapeutic intervals" stemming from large clinical trials within which to maintain their patients. The development and implementation of the INR (also endorsed by WHO) represents an example of ingenuity and a landmark in the history of anticoagulation. Unfortunately, very few realized that the INR was designed to harmonize PT results for patients on VKA and that its application in other conditions would have been questionable. As a matter of fact, the ISI required to convert PT ratios into INR is derived using plasma from patients on VKA. ${ }^{19}$ The coagulopathy induced by other clinical conditions (such as cirrhosis) may be different from that induced by VKA, thus making the ISI invalid, unless it is specifically determined for these conditions. This concept was not underscored for many years, ${ }^{20}$ and the MELD score was developed and validated by using the INR to express PT results in patients with cirrhosis. $^{21}$ The logical consequence is that PT results between laboratories are not harmonized and any given patient awaiting liver transplantation might receive a different PT (and therefore MELD) result depending on the thromboplastin used for testing. This situation might considerably modify the order of priority in the waiting list for transplantation ${ }^{18}$ and should therefore be urgently corrected. Proposals for a modified standardization model using plasma from patients with cirrhosis to calculate the ISI for commercial thromboplastin and the INR (called $\mathrm{INR}_{\text {liver }}$ as opposed to $\mathrm{INR}_{\mathrm{VKA}}$ ) have been made by two independent groups and wait to be implemented. 22,23

\section{Hypercoagulability and Cirrhosis}

As mentioned, hemostasis in cirrhosis is rebalanced, but the balance between the pro- and anticoagulant drivers is not that stable as in healthy subjects. While in healthy subjects there is a large excess of pro- and anticoagulants as well as platelets that stabilize the system, in patients with cirrhosis, there is a relative deficit in all of them. Therefore, it is logical to assume that the hemostatic balance is unstable and may tip toward hemorrhage or thrombosis depending on circumstantial risk factors and/or other conditions that are associated with cirrhosis (i.e., portal hypertension, bacterial infections, endothelial dysfunction, renal failure, etc.). As a matter of fact, patients with cirrhosis may present with thrombotic events, thus questioning the old belief that these patients are autoanticoagulated because of their hemostatic derangement. For instance, it was recognized many years ago that hospitalized patients with cirrhosis are not fully protected from venous thrombosis, including deep vein thrombosis and pulmonary embolism. ${ }^{24}$ In addition, a nationwide population-based case-control study performed in a large population of patients with liver disease and control subjects showed that these patients are cumulatively exposed to a relative risk of venous thromboembolism that amounted to nearly two times if compared with that of the control population. ${ }^{25}$ Furthermore, portal vein thrombosis (PVT) is considered as one of the most severe complications of cirrhosis resulting in portal hypertension and its complications such as variceal bleeding, thrombocytopenia, and splenomegaly. ${ }^{26}$ The frequency of PVT in patients with cirrhosis has been estimated to vary from approximately $1 \%$ among patients with compensated cirrhosis but 8 to $25 \%$ among those who are candidates for liver transplantation. ${ }^{26}$ Although PVT is not a contraindication for transplantation, it may worsen posttransplant prognosis, making patients awaiting transplantation candidates for primary prevention.

Recently, a randomized controlled trial showed that patients with end-stage chronic liver disease, who received daily prophylactic doses of low-molecular-weight heparin (LMWH) had less PVT and no more hemorrhagic events than those randomized to the usual care. In addition, the study showed that LMWH was effective in slowing the progression to decompensation of cirrhosis. ${ }^{27}$ As reduced flow velocity, plasma procoagulant imbalance and vessel-wall abnormalities $^{25}$ (Virchow triad) are mechanistic factors for venous thromboembolism, it is logical to assume that some sort of antithrombotic therapy (until recently strongly contraindicated in these patients) is justified.

The question is about whether it is possible to stratify patients based on their risk and make decisions on who should be given prophylaxis. This could be achieved on clinical grounds (including history of thrombosis, flow velocity in the portal system, etc.), but also by exploring the possible contribution of the blood hypercoagulability as measured by the laboratory. Recently, it was shown that thrombin generation measured in the presence versus the absence of thrombomodulin would allow to express results as the ratio (with/without thrombomodulin) and that this ratio is much more sensitive to detect signs of hypercoagulability than any other coagulation test. ${ }^{28}$ This phenomenon has been ascribed to an intrinsic resistance to the anticoagulant action of thrombomodulin that is characteristic not only of cirrhosis, ${ }^{28}$ but also of other noncirrhotic conditions associated with an increased risk of venous thromboembolism. ${ }^{29}$ By definition, the higher the ratio the higher the hypercoagulability. The study showed that ratios in a population of compensated cirrhosis were higher the normal and progressed from child A-B to $C$. The ratio was directly correlated with the increased levels of factor VIII (a typical feature in cirrhosis) ${ }^{28}$ and inversely with the decreasing levels of protein $C$ (another feature of cirrhosis). ${ }^{28}$ The thrombin generation (with/ without thrombomodulin) ratio was also correlated with the ratio between factor VIII and protein $\mathrm{C}^{28}$ Interestingly, (activated) protein $\mathrm{C}$ is the inhibitor of activated FVIII, thus making their ratio an index of hypercoagulability.

Finally, a recent study showed that in vitro supplementation of protein $C$ in plasma from patients with cirrhosis greatly reduces hypercoagulability. ${ }^{30}$ Hypercoagulability in 
cirrhosis was independently confirmed by Lisman et al $^{31}$ and Gatt et al. $^{32}$ The possibility that the thrombin generation (with/without thrombomodulin) ratio represents an in vivo index of hypercoagulability and therefore a thrombotic risk is being explored. Preliminary results showed that those patients with ratios higher than the 95th percentile of the distribution of healthy subjects have an increased risk to develop PVT (Dr. V. Lamura, unpublished data, 2015). In addition to the increased risk of thrombosis, hypercoagulability may contribute to the progression of liver fibrosis through parenchymal extinction. ${ }^{33}$

\section{Concluding Remarks}

The concomitant reduction of the pro- and anticoagulant drivers and the increased levels of von Willebrand factor make cirrhosis a clinical condition characterized by a rebalanced global hemostasis, despite the fact that the coagulation tests PT and aPTT are prolonged and that these patients are variably thrombocytopenic. Although the PT and aPTT are still used as predictors of bleeding and to guide transfusion with fresh frozen plasma or prohemostatic agents, they do not represent the coagulation mechanism that operates in vivo. There are pathophysiological and clinical considerations that explain this apparent paradox. Plasma in the PT or aPTT starts to clot few seconds after activation of coagulation; furthermore, the two tests are run in plasma in the absence of two of the key physiological activators of the protein $C$ and antithrombin systems (i.e., the endothelial cell receptor, thrombomodulin, and the heparin-like substances, located on the surface of endothelial cells). This makes PT and aPTT to be fully responsive to the procoagulants, but much less to the anticoagulants. The clinical considerations that support the unsuitability of the PT and aPTT in the cirrhosis rest on the evidence stemming from the literature (reviewed in Tripodi and Mannucci ${ }^{12}$ ) and clinical practice that the abnormalities of the two tests are poorly associated with the occurrence of bleeding events. It is therefore conceivable that the hemostasis derangement does not account for the bleeding events that occasionally occur in these patients.

Other conditions associated with cirrhosis (i.e., hemodynamic alterations subsequent to portal hypertension, bacterial infections, endothelial dysfunction, and renal failure) probably play a major role in determining bleeding. Effort to treat these conditions would probably be much more effective in preventing or controlling bleeding than the infusion of plasma or prohemostatic agents.

\section{References}

1 Tripodi A. Hemostasis abnormalities in chronic liver failure. In: Gines P, Kamath PS, Arroyo V, eds. Chronic Liver Failure. New York, NY: Springer Science + Business Media; 2011:289-303

2 Giannini EG, Savarino V. Thrombocytopenia in liver disease. Curr Opin Hematol 2008;15(5):473-480

3 Rockey DC, Caldwell SH, Goodman ZD, Nelson RC, Smith AD; American Association for the Study of Liver Diseases. Liver biopsy. Hepatology 2009;49(3):1017-1044
4 Lisman T, Bongers TN, Adelmeijer J, et al. Elevated levels of von Willebrand Factor in cirrhosis support platelet adhesion despite reduced functional capacity. Hepatology 2006;44(1):53-61

5 Tripodi A, Primignani M, Chantarangkul V, et al. Thrombin generation in patients with cirrhosis: the role of platelets. Hepatology 2006;44(2):440-445

6 Tripodi A, Primignani M, Chantarangkul V, et al. Global hemostasis tests in patients with cirrhosis before and after prophylactic platelet transfusion. Liver Int 2013;33(3):362-367

7 Afdhal NH, Giannini EG, Tayyab G, et al; ELEVATE Study Group. Eltrombopag before procedures in patients with cirrhosis and thrombocytopenia. N Engl J Med 2012;367(8):716-724

8 Tripodi A, Primignani M. Nontransfusional approach to increased platelet count in patients with cirrhosis and thrombocytopenia. Hepatology 2013;58(3):1177-1180

9 Lisman T, Leebeek FWG, de Groot PG. Haemostatic abnormalities in patients with liver disease. J Hepatol 2002;37(2):280-287

10 Tripodi A, Salerno F, Chantarangkul V, et al. Evidence of normal thrombin generation in cirrhosis despite abnormal conventional coagulation tests. Hepatology 2005;41(3):553-558

11 Dahlbäck B. Progress in the understanding of the protein $C$ anticoagulant pathway. Int J Hematol 2004;79(2):109-116

12 Tripodi A, Mannucci PM. The coagulopathy of chronic liver disease. N Engl J Med 2011;365(2):147-156

13 Bosch J, Thabut D, Bendtsen F, et al; European Study Group on rFVIIa in UGI Haemorrhage. Recombinant factor VIIa for upper gastrointestinal bleeding in patients with cirrhosis: a randomized, double-blind trial. Gastroenterology 2004;127(4):1123-1130

14 Bosch J, Thabut D, Albillos A, et al; International Study Group on rFVIIa in UGI Hemorrhage. Recombinant factor VIIa for variceal bleeding in patients with advanced cirrhosis: A randomized, controlled trial. Hepatology 2008;47(5):1604-1614

15 Lodge JP, Jonas S, Jones RM, et al; rFVIIa OLT Study Group. Efficacy and safety of repeated perioperative doses of recombinant factor VIIa in liver transplantation. Liver Transpl 2005;11(8):973-979

16 Planinsic RM, van der Meer J, Testa G, et al. Safety and efficacy of a single bolus administration of recombinant factor VIIa in liver transplantation due to chronic liver disease. Liver Transpl 2005; 11(8):895-900

17 Tripodi A, Chantarangkul V, Mannucci PM. Acquired coagulation disorders: revisited using global coagulation/anticoagulation testing. Br J Haematol 2009;147(1):77-82

18 Tripodi A, Chantarangkul V, Primignani M, et al. Thrombin generation in plasma from patients with cirrhosis supplemented with normal plasma: considerations on the efficacy of treatment with fresh-frozen plasma. Intern Emerg Med 2012; 7(2):139-144

19 Tripodi A, Chantarangkul V, Mannucci PM. The international normalized ratio to prioritize patients for liver transplantation: problems and possible solutions. J Thromb Haemost 2008;6(2): 243-248

20 Trotter JF, Olson J, Lefkowitz J, Smith AD, Arjal R, Kenison J. Changes in international normalized ratio (INR) and model for endstage liver disease (MELD) based on selection of clinical laboratory. Am J Transplant 2007;7(6):1624-1628

21 Malinchoc M, Kamath PS, Gordon FD, Peine CJ, Rank J, ter Borg PC. A model to predict poor survival in patients undergoing transjugular intrahepatic portosystemic shunts. Hepatology 2000; 31(4):864-871

22 Tripodi A, Chantarangkul V, Primignani M, et al. The international normalized ratio calibrated for cirrhosis (INR(liver)) normalizes prothrombin time results for model for end-stage liver disease calculation. Hepatology 2007;46(2):520-527

23 Bellest L, Eschwège V, Poupon R, Chazouillères $\mathrm{O}$, Robert $\mathrm{A}$. A modified international normalized ratio as an effective way of prothrombin time standardization in hepatology. Hepatology 2007;46(2):528-534 
24 Northup PG, McMahon MM, Ruhl AP, et al. Coagulopathy does not fully protect hospitalized cirrhosis patients from peripheral venous thromboembolism. Am J Gastroenterol 2006;101(7): 1524-1528, quiz 1680

25 Søgaard KK, Horváth-Puhó E, Grønbaek H, Jepsen P, Vilstrup H, Sørensen HT. Risk of venous thromboembolism in patients with liver disease: a nationwide population-based case-control study. Am J Gastroenterol 2009;104(1):96-101

26 Primignani M. Portal vein thrombosis, revisited. Dig Liver Dis 2010;42(3):163-170

27 Villa E, Cammà C, Marietta M, et al. Enoxaparin prevents portal vein thrombosis and liver decompensation in patients with advanced cirrhosis. Gastroenterology 2012;143(5):1253-60. e1, 4

28 Tripodi A, Primignani M, Chantarangkul V, et al. An imbalance of pro- vs anti-coagulation factors in plasma from patients with cirrhosis. Gastroenterology 2009;137(6):2105-2111
29 Dargaud Y, Trzeciak MC, Bordet JC, Ninet J, Negrier C. Use of calibrated automated thrombinography $+/$ - thrombomodulin to recognise the prothrombotic phenotype. Thromb Haemost 2006; 96(5):562-567

30 Tripodi A, Primignani M, Lemma L, Chantarangkul V, Mannucci PM. Evidence that low protein $C$ contributes to the procoagulant imbalance in cirrhosis. J Hepatol 2013;59(2):265-270

31 Lisman T, Bakhtiari K, Pereboom IT, Hendriks HG, Meijers JC, Porte RJ. Normal to increased thrombin generation in patients undergoing liver transplantation despite prolonged conventional coagulation tests. J Hepatol 2010;52(3):355-361

32 Gatt A, Riddell A, Calvaruso V, Tuddenham EG, Makris M, Burroughs AK. Enhanced thrombin generation in patients with cirrhosisinduced coagulopathy. J Thromb Haemost 2010;8(9):1994-2000

33 Tripodi A, Anstee QM, Sogaard KK, Primignani M, Valla DC. Hypercoagulability in cirrhosis: causes and consequences. J Thromb Haemost 2011;9(9):1713-1723 